\title{
Trends in burden of multidrug-resistant tuberculosis in countries, regions, and worldwide from 1990 to 2017: results from the Global Burden of Disease study
}

\author{
Ze-Jin Ou ${ }^{1 \dagger}$, Dan-Feng Yu ${ }^{2 \dagger}$, Yuan-Hao Liang ${ }^{1}$, Wen-Qiao He${ }^{1}$, Yong-Zhi Li ${ }^{1}$, Ya-Xian Meng ${ }^{1}$, Hu-Sheng Xiong ${ }^{1}$,
} Min-Yi Zhang ${ }^{1}$, Huan He ${ }^{1}$, Yu-Han Gao ${ }^{1}$, Fei Wu ${ }^{1}$ and Qing Chen ${ }^{1 *}$ (1)

\begin{abstract}
Background: Antituberculosis-drug resistance is an important public health issue, and its epidemiological patterns has dramatically changed in recent decades. This study aimed to estimate the trends of multidrug-resistant tuberculosis (MDR-TB), which can be used to inform health strategies.

Methods: Data were collected from the Global Burden of Disease study 2017. The estimated annual percentage changes (EAPCS) were calculated to assess the trends of MDR-TB burden at global, regional, and national level from 1990 to 2017 using the linear regression model.

Results: Globally, the age-standardized rate (ASR) of MDR-TB burden including incidence, prevalence, death and disability-adjusted life years (DALYs) had pronounced increasing trends from 1990 to 1999, with the EAPCs were 17.63 [95\% confidence interval (Cl): 10.77-24.92], 17.57 (95\% Cl 11.51-23.95), 21.21 (95\% Cl 15.96-26.69), and 21.90 (95\% Cl 16.55-27.50), respectively. Particularly, the largest increasing trends were seen in areas and countries with low and low-middle sociodemographic index (SDI). However, the trends in incidence, prevalence, death and DALYS of MDR-TB decreased globally from 2000 to 2017, with the respective EAPCs were -1.37 (95\% Cl-1.62 to - 1.12), -1.32 (95\% $C l-1.38$ to -1.26$)$, $-3.30(95 \% C l-3.56$ to -3.04$)$ and -3.32 (95\% Cl-3.59 to -3.06$)$. Decreasing trends of MDRTB were observed in most regions and countries, particularly that of death and DALYs in Slovenia were - 18.96 (95\% $\mathrm{Cl}-20.82$ to -17.06$)$ and $-19.35(95 \% \mathrm{Cl}-21.10$ to -17.55$)$, respectively. Whereas the pronounced increasing trends of MDR-TB occurred in Papua New Guinea, Singapore, and Australia.

Conclusions: The ASR of MDR-TB showed pronounced decreasing trends from 2000 to 2017. However, the MDR-TB burden remains a substantial challenge to the TB control globally, and requires effective control strategies and healthcare systems.
\end{abstract}

Keywords: Multidrug-resistant tuberculosis, Global burden of disease, Age-standardized rate, Estimated annual percentage change, Epidemiological trend

*Correspondence: qch.2009@163.com

${ }^{\dagger}$ Ze-Jin Ou and Dan-Feng Yu contributed equally to this work ${ }^{1}$ Guangdong Provincial Key Laboratory of Tropical Disease Research Department of Epidemiology, School of Public Health, Southern Medical University, 1838 Guangzhou North Road, Guangzhou 510515, China

Full list of author information is available at the end of the article

\section{Background}

Resistance to anti-tuberculosis (TB) drugs has been an increasing challenge to global TB control in recent years $[1,2]$. Multidrug-resistant tuberculosis (MDR-TB) is the major part of drug-resistant tuberculosis [3], and its original author(s) and the source, provide a link to the Creative Commons licence, and indicate if changes were made. The images or other third party material in this article are included in the article's Creative Commons licence, unless indicated otherwise in a credit line to the material. If material is not included in the article's Creative Commons licence and your intended use is not permitted by statutory regulation or exceeds the permitted use, you will need to obtain permission directly from the copyright holder. To view a copy of this licence, visit http://creativecommons.org/licenses/by/4.0/. The Creative Commons Public Domain Dedication waiver (http://creativeco mmons.org/publicdomain/zero/1.0/) applies to the data made available in this article, unless otherwise stated in a credit line to the data. 
trends are an important reference to the strategies of $\mathrm{TB}$ control.

MDR-TB is defined as the Mycobacterium tuberculosis that is resistant to isoniazid and rifampicin, which is strongly related to previous TB disease and its treatment $[4,5]$. In the 1990s, MDR-TB outbreaks were reported in the USA and Europe [6, 7], and global surveillance of antituberculosis drug resistance was considered urgent and necessary. In 1994, a global program on surveillance of drug-resistant tuberculosis was launched by the World Health Organization (WHO), and the results showed that MDR-TB had increased dramatically worldwide $[8$, 9]. During 1994 and 1997, the global prevalence of MDRTB was $1.4 \%$ in new TB patients, and $13 \%$ for TB patients treated previously [10]. It was reported that global MDRTB occurred in an estimated 460,000 cases and resulted in 230,000 deaths in 2017, and accounted for $3.6 \%$ of all new cases and $17 \%$ of treated cases [11]. The highest burden of MDR-TB has been reported to be in China, India, Russia, and South Africa, and those countries have $>60 \%$ of all cases worldwide [12]. Meanwhile, the increasing MDR-TB has elicited problems with decreasing cure rates and survival times [13]. Furthermore, the MDR-TB prevalence has soaked up $47 \%$ of the expense for the response to anti-TB programs globally [14], and strained local health resources $[8,15]$. These alarming data showed the urgent need for MDR-TB control.

The WHO set a "End TB" strategy based on a 90\% decrease in the TB incidence documented in 2015 to be reached by 2035 [16]. More efficient strategies and increasing investment and commitment to MDR-TB control will be needed [17]. The Global Burden of Disease (GBD) study had provided a methodological and conceptual framework to quantify health loss, which facilitates the assessment of progress and challenges in the MDR-TB control. Here, we estimated trends in the MDR-TB burden from 1990 to 2017 using data derived from the GBD 2017, which would facilitate the improvement of strategies for TB control.

\section{Materials and methods}

\section{Data source}

The data of MDR-TB were acquired from the Global Burden of Disease (GBD) study 2017 with the Global Health Data Exchange query tool (http://ghdx.healthdata .org/gbd-results-tool). According to the GBD online tools instruction, the number and rate of MDR-TB incidence, prevalence, death, and disability-adjusted life years (DALYs) were extracted based on age, sex, sociodemographic index (SDI) area, geographic region, and country, without any inclusion/exclusion criteria. Age was stratified into five groups: $<5,5-14,15-49,50-69$, and $>70$ years. The SDI areas were categorized into five levels: "low", "low-middle", "middle", "high-middle", and "high". According to geographic factor, the world was divided into 21 geographic regions, and 195 countries/ territories. The human development index (HDI) reflects the level of human development, and the availability of health resources in regions and countries. The data of HDI at national level were obtained from the United Nations Development Program (http://hdr.undp.org/en/ data).

\section{Statistical analyses}

The age-standardized rate (ASR) is a critical and representative parameter when considering differences in the age structure of multiple populations. The ASR is a weighted mean of age-specific rates. The ASR is calculated on basis of the age structure of standard populations using the following formula:

$$
\mathrm{ASR}=\frac{\sum_{i=1}^{A} a_{i} w_{i}}{\sum_{i=1}^{A} w_{i}} \times 100,000
$$

where $a_{i}$ is the age-specific rate in the $i^{\text {th }}$ age group, $w_{i}$ is the number of persons (or the weight) in the corresponding $i^{\text {th }}$ age subgroup of the selected reference standard population, and A is the number of age groups.

The ASR trend is a widely accepted measure that estimates the changing patterns of disease burden. The estimated annual percentage change (EAPC) is a common index reflecting the temporal trend of ASR, which has been described in detail elsewhere [18]. A regression line was fitted to the natural logarithm of the ASR. EAPC and its $95 \%$ confidence interval $(C I)$ were estimated with the linear regression model. The formula is as following:

$$
\begin{aligned}
& \mathrm{y}=\alpha+\beta \mathrm{x}+\varepsilon \\
& \mathrm{EAPC}=100 \times(\exp (\beta)-1)
\end{aligned}
$$

where $y=\ln$ (ASR), and $\mathrm{x}$ is the calendar year. The trends were judged: if the EAPC and its 95\% CI were $>0$, the ASR was in an increasing trend; if EAPC and its $95 \% C I$ were $<0$, the ASR was in a decreasing trend; other values meant that the ASR was stable over time. For the aim to analyze the impact factors of EAPC, the respective associations between EAPCs and ASR in 2000, and between EAPCs and HDI in 2017, were calculated by using the Pearson correlation analysis in the Statistical Package for Social Sciences (SPSS; version 25.0; SPSS Inc., Chicago, IL, USA). The choropleth maps were drawn using the statistical software $\mathrm{R}$ 3.6.2 (Lucent Technologies, Jasmine Mountain, USA). A $P$ value of less than 0.05 was deemed to be statistically significant. 


\section{Results}

Trends in MDR-TB incidence

During 1990 and 1999, the incidence of MDR-TB had a pronounced rising trend globally, with the overall age-standardized incidence rate (ASIR) increasing by an annual average of $17.63 \%(\mathrm{EAPC}=17.63,95 \% \mathrm{CI}$ 10.77-24.92). The upward trends of ASIR occurred in all SDI areas and geographic regions, expect high-income North America (Table 1; Fig. 1a). At national level, the ASIR of MDR-TB showed increasing trends in 186 countries/territories, particularly Turkmenistan, Somalia, and Kyrgyzstan, with the EAPCs were 66.78 (95\% CI 55.50-78.89), 66.08 (95\% CI 55.93-76.89), and 63.94 (95\% CI 51.99-76.84), respectively. However, the downward trends of ASIR were observed only in United States $(\mathrm{EAPC}=-6.78,95 \% C I-8.19$ to -5.36$)$ (Additional file 1: Table S4; Figure S7A-C).

Globally, the incident number of MDR-TB was $432.70 \times 10^{3} \quad(95 \%$ uncertainty interval (UI): $254.61 \times 10^{3}-726.95 \times 10^{3}$ ) in 2017 , with an increase of $8.29 \%$ from 2000 to 2017. During 2000 and 2017, the ASIR had a decreasing trend (EAPC $=-1.37,95 \%$ $C I-1.62$ to -1.12 ) (Table 1; Fig. 1b). The downward trends of ASIR occured in SDI areas, expect low and low-middle SDI areas. In terms of 21 regions, the most pronounced decreasing trends were observed in East Asia and High-income Asia Pacific, in which EPACs were $-10.98(95 \% \quad C I-11.66$ to -10.29$)$ and -7.30 (95\%CI -8.70 to -5.87$)$. Whereas the largest increasing trends occurred in Oceania (EAPC $=9.53$, 95\% CI 7.9311.15) (Table 1; Figs. 1b, 2a-c). Among 195 countries/ territories, the ASIR showed downward trends in 112 countries, and the largest one was in Slovenia from 2000 to 2017 (EAPC $=-14.11,95 \% C I-15.36$ to -12.83$)$. On the other hand, the ASIR showed rising trends in 54 countries, particularly Papua New Guinea $(\mathrm{EAPC}=9.83$, 95\% CI 8.23-11.45), followed by Australia and Finland (Additional file 1: Table S5; Fig. 3a-c). The EAPCs (20002017) had a positive correlation with the ASIR in 2000 at a national level $(\rho=0.25, P<0.001)$ (Fig. 4a), but no with the HDI in 2017.

\section{Trends in MDR-TB prevalence}

Globally, the ASR of prevalence showed an obvious upward trend from 1990 to 1999, with the EAPC was 17.57 (95\% CI 11.51-23.95). In SDI areas and geographic regions, the ASR of prevalence had the similar trends to that of ASIR from 1990 to 1999 (Additional file 1: Table S1; Fig. 1a). With regard to national level, from 1990 to 1999 , the rising trends were observed in 185 countries/territories, and the largest one was Somalia $(\mathrm{EAPC}=67.92,95 \%$ CI 58.28-78.16), followed by Turkmenistan, and Kyrgyzstan. However, the trends of prevalence decreased only in United States, with the EAPC of - 10.84 (95\% CI - 11.40 to - 10.28) (Additional file 1: Table S4, Figure S8A-C ).

The number of MDR-TB prevalence was $464.12 \times 10^{3}$ $\left(95 \%\right.$ UI: $\left.229.12 \times 10^{3}-863.33 \times 10^{3}\right)$ worldwide in 2017 , with an increase of $9.92 \%$ from 2000 to 2017. During 2000 and 2017, the prevalence of MDR-TB had a downward trend globally, with the EAPC was -1.32 (95\% CI -1.38 to -1.26 ) (Additional file 1: Table S1; Fig. 1). The trends of prevalence rose in areas of low- and lowmiddle SDI, while decreasing in other areas. Among 21 geographical regions, the most pronounced downward trends were observed in High-income Asia Pacific (EAPC $=-8.77,95 \% C I-9.98$ to -7.55$)$, while the largest increasing trends occurred in Oceania and Australasia (Additional file 1: Table S1; Fig. 1b, and Additional file 1: Figure S1A-C). At national level, the decreasing trends of prevalence were observed in 104 countries from 2000 to 2017, particularly Slovenia (EAPC $=-13.69,95 \%$ $C I-15.04$ to -12.31$)$. While the rising trends occurred in 55 countries, and the largest one was in Papua New Guinea (EAPC $=9.28$, 95\% CI 7.81-10.78), followed by Finland and Australia (Additional file 1: Table S5; Figure S4A-C). The EAPCs (2000-2017) had a positive correlation with the ASR of prevalence in 2000 at a national level $(\rho=0.18, P=0.02$ ) (Fig. 4b), but no with the HDI in 2017.

\section{Trends in MDR-TB related death}

Globally, the age-standardized death rate (ASDR) of MDR-TB had a rising trend from 1990 to 1999 (EAPC $=21.21$, 95\% CI 15.96-26.69). During 1990 and 1999, the upward trends of ASDR were observed in all SDI areas and most geographic regions, whereas the decreasing trend occurred only in High-income North America (EAPC $=-15.91,95 \% C I-18.52$ to -13.22 ) (Additional file 1: Table S2; Fig. 1a). At national level, the upward trends were documented in 186 countries/ territories, and the largest increasing occurred in Somalia $(\mathrm{EAPC}=81.08,95 \%$ CI 72.54-90.05), followed by Kyrgyzstan, and Turkmenistan. However, the decreasing trends were seen in six countries, particularly United States, in which the EAPC was - 16.72 (95\% CI-19.41 to - 13.94) (Additional file 1: Table S4, Figure S9A-C).

The number of deaths attributed to MDR-TB was $126.89 \times 10^{3}\left(95 \%\right.$ UI: $\left.70.06 \times 10^{3}-202.17 \times 10^{3}\right)$ in 2017 , with a decrease of $10.17 \%$ from 2000 to 2017 . Globally, the ASDR of MDR-TB had a decreasing trend from 2000 to 2017 (EAPC $=-3.30,95 \% C I-3.56$ to -3.04$)$ (Additional file 1: Table S2; Fig. 1b). The ASDR had downward trends in all SDI areas and regions from 2000 to 2017, expect Oceania and Australasia. The most pronounced decreasing trends were documented in East Asia $\quad(\mathrm{EAPC}=-13.57, \quad 95 \% \quad C I-14.21$ to -12.92$)$, 
Table 1 the percentage changes in absolute number and EAPCs of MDR-TB incidence from 1990 to 2017 in global, sexes, SDI areas and geographic regions

\begin{tabular}{|c|c|c|c|c|c|c|c|c|}
\hline \multirow[b]{2}{*}{ Characteristics } & \multicolumn{2}{|l|}{$\underline{1999}$} & \multicolumn{2}{|l|}{$\underline{1990-1999}$} & \multicolumn{2}{|l|}{$\underline{2017}$} & \multicolumn{2}{|l|}{$\underline{2000-2017}$} \\
\hline & $\begin{array}{l}\text { Number } \\
\mathbf{x} 10^{\mathbf{3}}(95 \% \text { UI) }\end{array}$ & $\begin{array}{l}\text { ASR/100 } 000 \\
(95 \% \text { UI })\end{array}$ & $\begin{array}{l}\text { Change } \\
\text { in number } \\
(\%)\end{array}$ & $\begin{array}{l}\text { EAPC } \\
(95 \% \text { Cl) }\end{array}$ & $\begin{array}{l}\text { Number } \\
\times 10^{\mathbf{3}}(95 \% \text { UI) }\end{array}$ & $\begin{array}{l}\text { ASR/100 } 000 \\
(95 \% \text { UI })\end{array}$ & $\begin{array}{l}\text { Change in } \\
\text { number (\%) }\end{array}$ & $\begin{array}{l}\text { EAPC } \\
(95 \% \text { Cl) }\end{array}$ \\
\hline Overall & $\begin{array}{c}383.50 \\
(290.12-517.25)\end{array}$ & $\begin{array}{c}6.41 \\
(4.85-8.64)\end{array}$ & 558.06 & $\begin{array}{l}17.63 \\
(10.77-24.92)\end{array}$ & $\begin{array}{c}432.77 \\
(254.61-726.95)\end{array}$ & $\begin{array}{c}5.55 \\
(3.29-9.29)\end{array}$ & 8.29 & $\begin{array}{l}-1.37 \\
(-1.62 \text { to }-1.12)\end{array}$ \\
\hline \multicolumn{9}{|l|}{ Sex } \\
\hline Male & $\begin{array}{c}215.27 \\
(165.10-285.45)\end{array}$ & $\begin{array}{c}7.36 \\
(5.63-9.77)\end{array}$ & 575.03 & $\begin{array}{l}17.89 \\
(11.09-25.10)\end{array}$ & $\begin{array}{c}240.92 \\
(145.81-396.46)\end{array}$ & $\begin{array}{c}6.17 \\
(3.75-10.14)\end{array}$ & 7.13 & $\begin{array}{l}-1.58 \\
(-1.81 \text { to }-1.34)\end{array}$ \\
\hline Female & $\begin{array}{c}168.23 \\
(124.10-232.42)\end{array}$ & $\begin{array}{c}5.55 \\
(4.10-7.66)\end{array}$ & 537.55 & $\begin{array}{l}17.30 \\
(10.36-24.67)\end{array}$ & $\begin{array}{c}191.85 \\
(109.57-334.02)\end{array}$ & $\begin{array}{c}4.99 \\
(2.86-8.66)\end{array}$ & 9.80 & $\begin{array}{l}-1.13 \\
(-1.40 \text { to }-0.86)\end{array}$ \\
\hline \multicolumn{9}{|l|}{ SDI } \\
\hline Low & $\begin{array}{c}57.85 \\
(31.12-110.89)\end{array}$ & $\begin{array}{c}8.17 \\
(4.30-16.14)\end{array}$ & 2054.73 & $\begin{array}{l}32.73 \\
(24.72-41.25)\end{array}$ & $\begin{array}{c}121.88 \\
(60.13-247.13)\end{array}$ & $\begin{array}{c}11.23 \\
(5.28-23.28)\end{array}$ & 90.22 & $\begin{array}{l}1.08 \\
(0.85-1.30)\end{array}$ \\
\hline Low-middle & $\begin{array}{c}82.58 \\
(47.27-145.63)\end{array}$ & $\begin{array}{c}7.59 \\
(4.25-13.67)\end{array}$ & 1243.54 & $\begin{array}{l}26.40 \\
(18.82-34.46)\end{array}$ & $\begin{array}{c}145.89 \\
(70.89-263.95)\end{array}$ & $\begin{array}{c}9.32 \\
(4.44-17.28)\end{array}$ & 61.69 & $\begin{array}{l}0.47 \\
(0.11-0.84)\end{array}$ \\
\hline Middle & $\begin{array}{c}135.47 \\
(87.38-194.95)\end{array}$ & $\begin{array}{c}8.14 \\
(5.26-11.8)\end{array}$ & 366.99 & $\begin{array}{l}13.27 \\
(6.4-20.59)\end{array}$ & $\begin{array}{c}96.80 \\
(48.14-175.83)\end{array}$ & $\begin{array}{c}4.44 \\
(2.22-8.10)\end{array}$ & -28.73 & $\begin{array}{l}-3.90 \\
(-4.17 \\
\text { to }-3.63)\end{array}$ \\
\hline High-middle & $\begin{array}{c}98.64 \\
(66.20-143.87)\end{array}$ & $\begin{array}{c}7.90 \\
(5.26-11.56)\end{array}$ & 463.10 & $\begin{array}{l}15.79 \\
(8.51-23.55)\end{array}$ & $\begin{array}{c}64.68 \\
(42.19-96.12)\end{array}$ & $\begin{array}{c}4.12 \\
(2.70-6.14)\end{array}$ & -35.78 & $\begin{array}{l}-4.08 \\
(-4.63 \text { to }-3.54)\end{array}$ \\
\hline High & $\begin{array}{c}6.05 \\
(3.90-10.40)\end{array}$ & $\begin{array}{c}0.54 \\
(0.34-0.94)\end{array}$ & 183.49 & $\begin{array}{l}9.29 \\
(4.32-14.50)\end{array}$ & $\begin{array}{c}2.86 \\
(1.75-5.15)\end{array}$ & $\begin{array}{c}0.22 \\
(0.14-0.38)\end{array}$ & -52.36 & $\begin{array}{l}-6.25 \\
(-6.93 \text { to }-5.57)\end{array}$ \\
\hline \multicolumn{9}{|l|}{ Regions } \\
\hline East Asia & $\begin{array}{c}174.58 \\
(118.01-246.69)\end{array}$ & $\begin{array}{c}13.08 \\
(8.89-18.48)\end{array}$ & 272.72 & $\begin{array}{l}11.31 \\
(4.02-19.11)\end{array}$ & $\begin{array}{c}35.78 \\
(8.19-111.13)\end{array}$ & $\begin{array}{c}2.22 \\
(0.51-6.96)\end{array}$ & -78.58 & $\begin{array}{l}-10.98 \\
(-11.66 \\
\text { to }-10.29)\end{array}$ \\
\hline South Asia & $\begin{array}{c}88.80 \\
(32.12-200.49)\end{array}$ & $\begin{array}{c}7.82 \\
(2.84-17.74)\end{array}$ & 4328.87 & $\begin{array}{l}42.85 \\
(32.57-53.93)\end{array}$ & $\begin{array}{c}216.16 \\
(59.52-491.95)\end{array}$ & $\begin{array}{c}12.96 \\
(3.54-29.61)\end{array}$ & 112.02 & $\begin{array}{l}1.91 \\
(1.34-2.49)\end{array}$ \\
\hline Southeast Asia & $\begin{array}{c}18.74 \\
(9.13-47.79)\end{array}$ & $\begin{array}{c}4.01 \\
(1.98-10.04)\end{array}$ & 891.37 & $\begin{array}{l}21.07 \\
(11.74-31.17)\end{array}$ & $\begin{array}{c}20.14 \\
(11.97-32.22)\end{array}$ & $\begin{array}{c}3.11 \\
(1.85-4.94)\end{array}$ & 4.47 & $\begin{array}{l}-1.36 \\
(-1.60 \text { to }-1.13)\end{array}$ \\
\hline Central Asia & $\begin{array}{c}5.85 \\
(3.87-9.25)\end{array}$ & $\begin{array}{c}8.23 \\
(5.42-12.93)\end{array}$ & 6722.47 & $\begin{array}{l}48.26 \\
(33.64-64.48)\end{array}$ & $\begin{array}{c}15.00 \\
(11.24-19.68)\end{array}$ & $\begin{array}{c}16.14 \\
(12.07-21.23)\end{array}$ & 112.47 & $\begin{array}{l}1.87 \\
(-0.35-4.14)\end{array}$ \\
\hline $\begin{array}{l}\text { High-income } \\
\text { Asia Pacific }\end{array}$ & $\begin{array}{c}1.54 \\
(1.13-2.20)\end{array}$ & $\begin{array}{c}0.71 \\
(0.51-1.03)\end{array}$ & 254.03 & $\begin{array}{l}10.85 \\
(5.12-16.88)\end{array}$ & $\begin{array}{c}0.77 \\
(0.18-2.12)\end{array}$ & $\begin{array}{c}0.29 \\
(0.07-0.82)\end{array}$ & -50.42 & $\begin{array}{l}-7.30 \\
(-8.70 \text { to }-5.87)\end{array}$ \\
\hline Oceania & $\begin{array}{c}0.06 \\
(0.01-0.20)\end{array}$ & $\begin{array}{c}0.88 \\
(0.18-2.77)\end{array}$ & 1528.54 & $\begin{array}{l}28.75 \\
(21.89-36)\end{array}$ & $\begin{array}{c}0.57 \\
(0.29-1.00)\end{array}$ & $\begin{array}{c}4.80 \\
(2.44-8.35)\end{array}$ & 710.75 & $\begin{array}{l}9.53 \\
(7.93-11.15)\end{array}$ \\
\hline Australasia & $\begin{array}{c}0.01 \\
(0.005-0.02)\end{array}$ & $\begin{array}{c}0.05 \\
(0.02-0.10)\end{array}$ & 61.42 & $\begin{array}{l}3.86 \\
(1.31-6.47)\end{array}$ & $\begin{array}{c}0.04 \\
(0.03-0.07)\end{array}$ & $\begin{array}{c}0.15 \\
(0.09-0.24)\end{array}$ & 323.96 & $\begin{array}{l}7.54 \\
(7.09-8.00)\end{array}$ \\
\hline $\begin{array}{l}\text { Eastern } \\
\text { Europe }\end{array}$ & $\begin{array}{c}30.93 \\
(22.15-42.10)\end{array}$ & $\begin{array}{c}12.70 \\
(9.12-17.28)\end{array}$ & 1667.60 & $\begin{array}{l}31.15 \\
(21.59-41.46)\end{array}$ & $\begin{array}{c}41.35 \\
(27.21-57.89)\end{array}$ & $\begin{array}{c}17.64 \\
(11.54-24.59)\end{array}$ & 21.56 & $\begin{array}{l}1.41 \\
(0.16-2.66)\end{array}$ \\
\hline $\begin{array}{l}\text { Western } \\
\text { Europe }\end{array}$ & $\begin{array}{c}0.70 \\
(0.59-0.81)\end{array}$ & $\begin{array}{c}0.18 \\
(0.15-0.21)\end{array}$ & 144.69 & $\begin{array}{l}9.58 \\
(8.21-10.97)\end{array}$ & $\begin{array}{c}0.73 \\
(0.57-0.95)\end{array}$ & $\begin{array}{c}0.18 \\
(0.14-0.23)\end{array}$ & 0.56 & $\begin{array}{l}-1.01 \\
(-1.51 \text { to }-0.51)\end{array}$ \\
\hline Central Europe & $\begin{array}{c}0.79 \\
(0.53-1.30)\end{array}$ & $\begin{array}{c}0.60 \\
(0.39-0.97)\end{array}$ & 354.42 & $\begin{array}{l}14.17 \\
(6.6-22.27)\end{array}$ & $\begin{array}{c}0.58 \\
(0.39-0.82)\end{array}$ & $\begin{array}{c}0.45 \\
(0.29-0.63)\end{array}$ & -27.56 & $\begin{array}{l}-2.29 \\
(-3.28 \text { to }-1.29)\end{array}$ \\
\hline $\begin{array}{l}\text { High-income } \\
\text { North } \\
\text { America }\end{array}$ & $\begin{array}{c}0.23 \\
(0.19-0.28)\end{array}$ & $\begin{array}{c}0.07 \\
(0.05-0.08)\end{array}$ & -38.50 & $\begin{array}{l}-6.28 \\
(-7.68 \text { to }-4.86)\end{array}$ & $\begin{array}{c}0.12 \\
(0.08-0.18)\end{array}$ & $\begin{array}{c}0.03 \\
(0.02-0.05)\end{array}$ & -41.23 & $\begin{array}{l}-2.96 \\
(-3.33 \text { to }-2.59)\end{array}$ \\
\hline $\begin{array}{l}\text { Andean Latin } \\
\text { America }\end{array}$ & $\begin{array}{c}3.23 \\
(1.59-5.84)\end{array}$ & $\begin{array}{c}7.64 \\
(3.76-13.79)\end{array}$ & 773.25 & $\begin{array}{l}22.40 \\
(18.12-26.83)\end{array}$ & $\begin{array}{c}2.81 \\
(2.05-4.19)\end{array}$ & $\begin{array}{c}4.62 \\
(3.36-6.9)\end{array}$ & -18.48 & $\begin{array}{l}-3.98 \\
(-4.41 \text { to }-3.54)\end{array}$ \\
\hline $\begin{array}{l}\text { Central Latin } \\
\text { America }\end{array}$ & $\begin{array}{c}1.02 \\
(0.59-1.81)\end{array}$ & $\begin{array}{c}0.62 \\
(0.35-1.08)\end{array}$ & 2211.58 & $\begin{array}{l}31.07 \\
(20.52-42.54)\end{array}$ & $\begin{array}{c}1.42 \\
(0.66-2.92)\end{array}$ & $\begin{array}{c}0.56 \\
(0.26-1.15)\end{array}$ & 28.99 & $\begin{array}{l}-1.76 \\
(-2.35 \text { to }-1.16)\end{array}$ \\
\hline Caribbean & $\begin{array}{c}0.17 \\
(0.07-0.48)\end{array}$ & $\begin{array}{c}0.44 \\
(0.17-1.22)\end{array}$ & 167.35 & $\begin{array}{l}8.51 \\
(4.22-12.98)\end{array}$ & $\begin{array}{c}0.10 \\
(0.04-0.23)\end{array}$ & $\begin{array}{c}0.20 \\
(0.08-0.49)\end{array}$ & -44.12 & $\begin{array}{l}-5.79 \\
(-7.83 \text { to }-3.71)\end{array}$ \\
\hline $\begin{array}{c}\text { Tropical Latin } \\
\text { America }\end{array}$ & $\begin{array}{c}1.40 \\
(0.38-3.57)\end{array}$ & $\begin{array}{c}0.84 \\
(0.23-2.11)\end{array}$ & 5272.18 & $\begin{array}{l}48.39 \\
(39.68-57.64)\end{array}$ & $\begin{array}{c}2.12 \\
(0.48-5.78)\end{array}$ & $\begin{array}{c}0.90 \\
(0.21-2.46)\end{array}$ & 37.90 & $\begin{array}{l}-0.51 \\
(-1.42-0.41)\end{array}$ \\
\hline
\end{tabular}


Table 1 (continued)

\begin{tabular}{|c|c|c|c|c|c|c|c|c|}
\hline \multirow[b]{2}{*}{ Characteristics } & \multicolumn{2}{|l|}{$\underline{1999}$} & \multicolumn{2}{|l|}{$\underline{1990-1999}$} & \multicolumn{2}{|l|}{$\underline{2017}$} & \multicolumn{2}{|l|}{$\underline{2000-2017}$} \\
\hline & $\begin{array}{l}\text { Number } \\
\mathbf{x} 10^{\mathbf{3}}(95 \% \text { UI) }\end{array}$ & $\begin{array}{l}\text { ASR/100 } 000 \\
(95 \% \text { UI })\end{array}$ & $\begin{array}{l}\text { Change } \\
\text { in number } \\
(\%)\end{array}$ & $\begin{array}{l}\text { EAPC } \\
(95 \% \text { Cl) }\end{array}$ & $\begin{array}{l}\text { Number } \\
\times 10^{\mathbf{3}}(95 \% \text { UI) }\end{array}$ & $\begin{array}{l}\text { ASR/100 } 000 \\
(95 \% \text { UI })\end{array}$ & $\begin{array}{l}\text { Change in } \\
\text { number (\%) }\end{array}$ & $\begin{array}{l}\text { EAPC } \\
(95 \% \text { Cl) }\end{array}$ \\
\hline $\begin{array}{l}\text { Southern Latin } \\
\text { America }\end{array}$ & $\begin{array}{c}0.17 \\
(0.10-0.30)\end{array}$ & $\begin{array}{c}0.32 \\
(0.18-0.54)\end{array}$ & 511.06 & $\begin{array}{l}19.46 \\
(16.21-22.79)\end{array}$ & $\begin{array}{c}0.11 \\
(0.03-0.32)\end{array}$ & $\begin{array}{c}0.16 \\
(0.05-0.49)\end{array}$ & -40.47 & $\begin{array}{l}-5.83 \\
(-6.87 \text { to }-4.79)\end{array}$ \\
\hline $\begin{array}{l}\text { Eastern Sub- } \\
\text { Saharan } \\
\text { Africa }\end{array}$ & $\begin{array}{c}17.55 \\
(10.87-28.72)\end{array}$ & $\begin{array}{c}8.75 \\
(5.23-14.98)\end{array}$ & 3920.28 & $\begin{array}{l}40.07 \\
(29.48-51.53)\end{array}$ & $\begin{array}{c}34.27 \\
(21.73-53.29)\end{array}$ & $\begin{array}{c}9.62 \\
(6.20-14.99)\end{array}$ & 78.79 & $\begin{array}{l}-0.17 \\
(-0.70-0.35)\end{array}$ \\
\hline $\begin{array}{l}\text { Southern } \\
\text { Sub-Saharan } \\
\text { Africa }\end{array}$ & $\begin{array}{c}8.03 \\
(4.20-17.60)\end{array}$ & $\begin{array}{c}12.38 \\
(6.47-27.75)\end{array}$ & 707.05 & $\begin{array}{l}23.11 \\
(22.54-23.67)\end{array}$ & $\begin{array}{c}11.15 \\
(6.21-20.66)\end{array}$ & $\begin{array}{c}13.79 \\
(7.65-25.24)\end{array}$ & 20.43 & $\begin{array}{l}-0.46 \\
(-1.86-0.96)\end{array}$ \\
\hline $\begin{array}{l}\text { Western } \\
\text { Sub-Saharan } \\
\text { Africa }\end{array}$ & $\begin{array}{c}17.21 \\
(7.77-38.43)\end{array}$ & $\begin{array}{c}8.32 \\
(3.76-18.33)\end{array}$ & 1164.55 & $\begin{array}{l}24.50 \\
(17.01-32.47)\end{array}$ & $\begin{array}{c}30.44 \\
(13.54-64.69)\end{array}$ & $\begin{array}{c}8.59 \\
(3.83-18.26)\end{array}$ & 62.28 & $\begin{array}{l}-0.48 \\
(-1.02-0.06)\end{array}$ \\
\hline $\begin{array}{l}\text { North Africa } \\
\text { and Middle } \\
\text { East }\end{array}$ & $\begin{array}{c}6.76 \\
(4.11-10.36)\end{array}$ & $\begin{array}{c}1.74 \\
(1.06-2.67)\end{array}$ & 1760.45 & $\begin{array}{l}33.46 \\
(28.65-38.46)\end{array}$ & $\begin{array}{c}7.10 \\
(4.65-11.81)\end{array}$ & $\begin{array}{c}1.21 \\
(0.8-2.00)\end{array}$ & -2.84 & $\begin{array}{l}-2.56 \\
(-2.73 \text { to }-2.39)\end{array}$ \\
\hline $\begin{array}{l}\text { Central Sub- } \\
\text { Saharan } \\
\text { Africa }\end{array}$ & $\begin{array}{c}5.72 \\
(1.73-16.90)\end{array}$ & $\begin{array}{c}10.17 \\
(3.12-29.55)\end{array}$ & 673.46 & $\begin{array}{l}18.11 \\
(10.74-25.97)\end{array}$ & $\begin{array}{c}12.01 \\
(2.87-32.58)\end{array}$ & $\begin{array}{c}12.33 \\
(2.97-34.12)\end{array}$ & 99.63 & $\begin{array}{l}0.65 \\
(0.45-0.85)\end{array}$ \\
\hline
\end{tabular}

MDR-TB: Multidrug resistant tuberculosis; EAPC: Estimated annual percentage change; ASR: Age-standardized rate; Cl Confidence interval; UI: uncertainty interval; SDI: Socio-demographic index

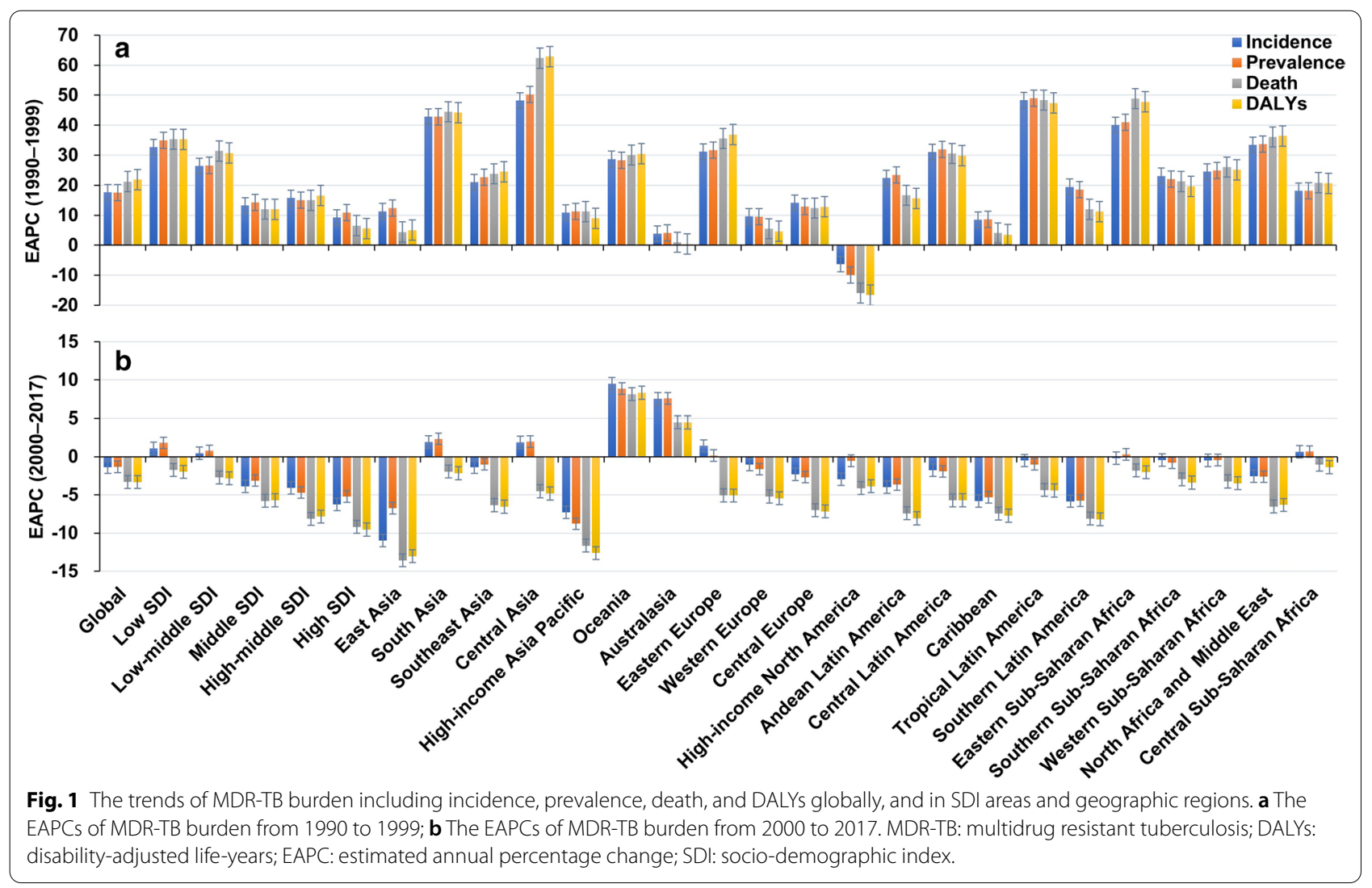



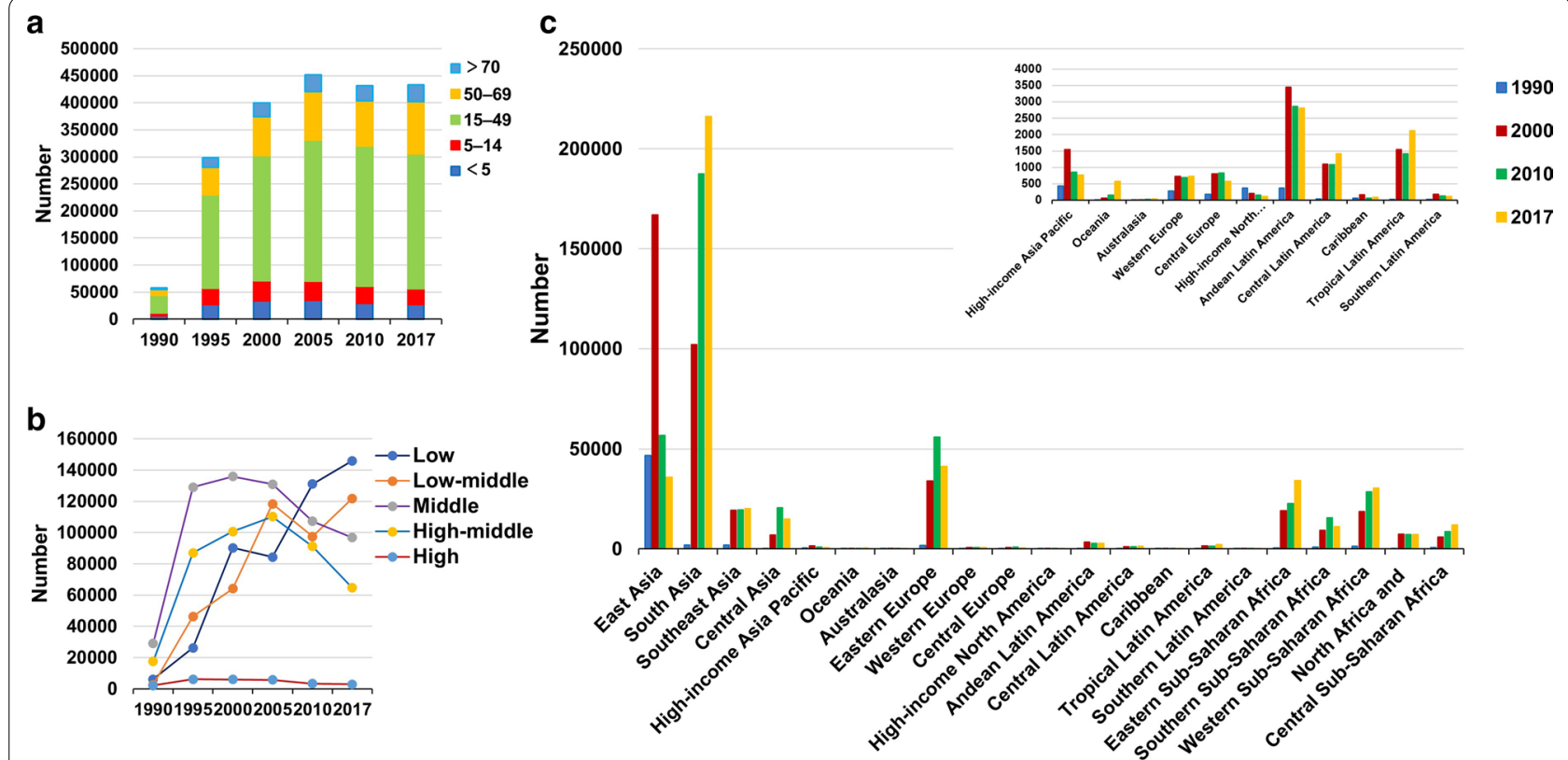

Fig. 2 The distribution of the incident number of MDR-TB globally, and in SDI areas and geographic regions from1990 to 2017. a The incident number of MDR-TB in age groups; $\mathbf{b}$ The changing of incident number of MDR-TB in SDI areas; $\mathbf{c}$ The incident number of MDR-TB in geographical regions. MDR-TB: multidrug resistant tuberculosis; SDI: socio-demographic index

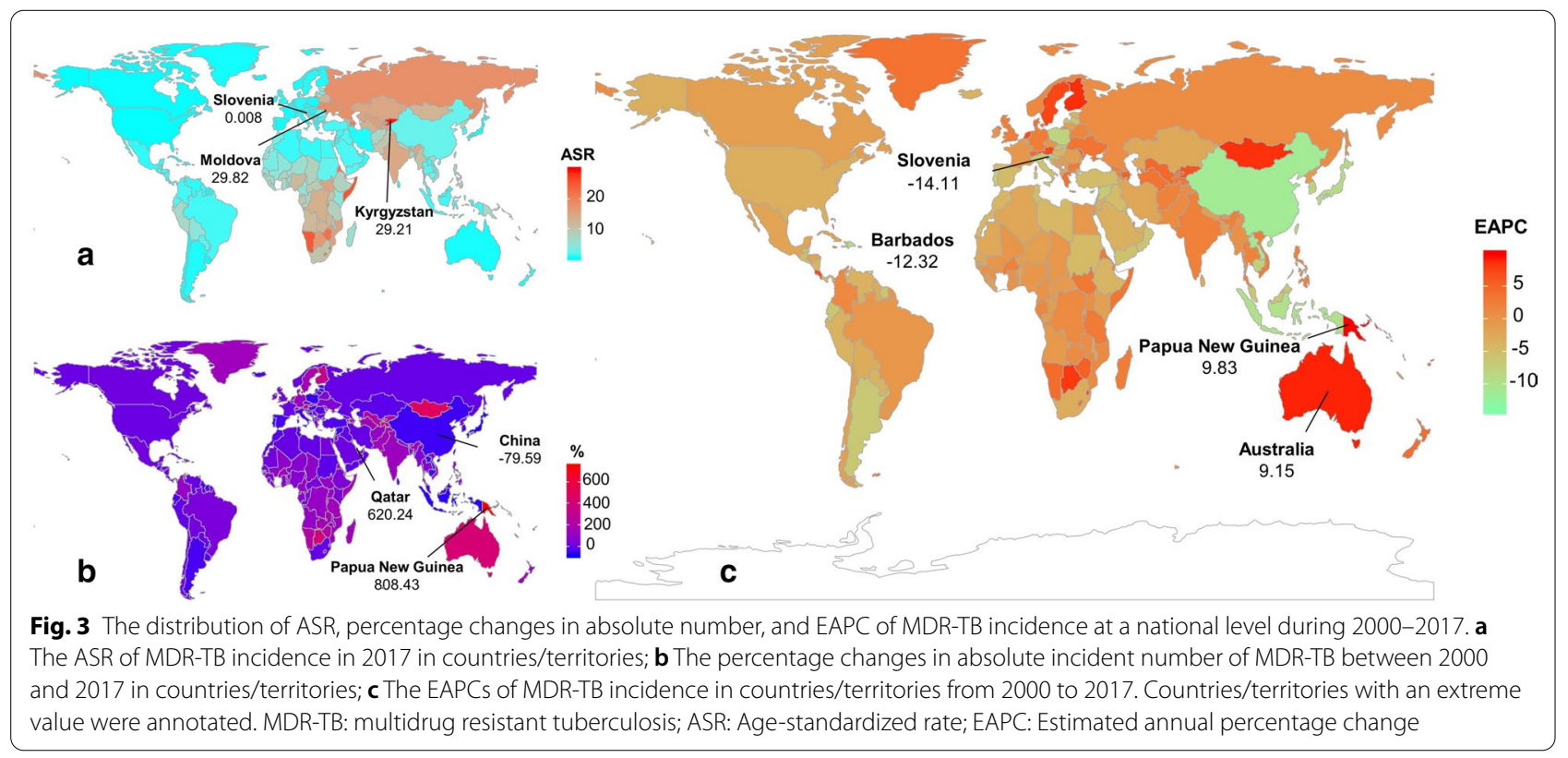

followed by high-income Asia Pacific and Southern Latin America (Additional file 1: Table S2; Fig. 1b, and Additional file 1: Figure S2A-C). At national level, the decreasing trends were documented in 164 countries/territories from 2000 to 2017, and the largest one was in Slovenia $(\mathrm{EAPC}=-18.96,95 \% \mathrm{CI}-20.82$ to -17.06$)$, followed by Maldives, and Laos. On the other hand, the upward trends of ASDR were observed in seventeen countries, particularly Singapore $(\mathrm{EAPC}=12.67,95 \%$ CI 10.60 14.78) (Additional file 1: Table S5; Figure S5A-C). The EAPCs (2000-2017) had a positive correlation with the ASDR in 2000, and a negative correlation with the HDI 

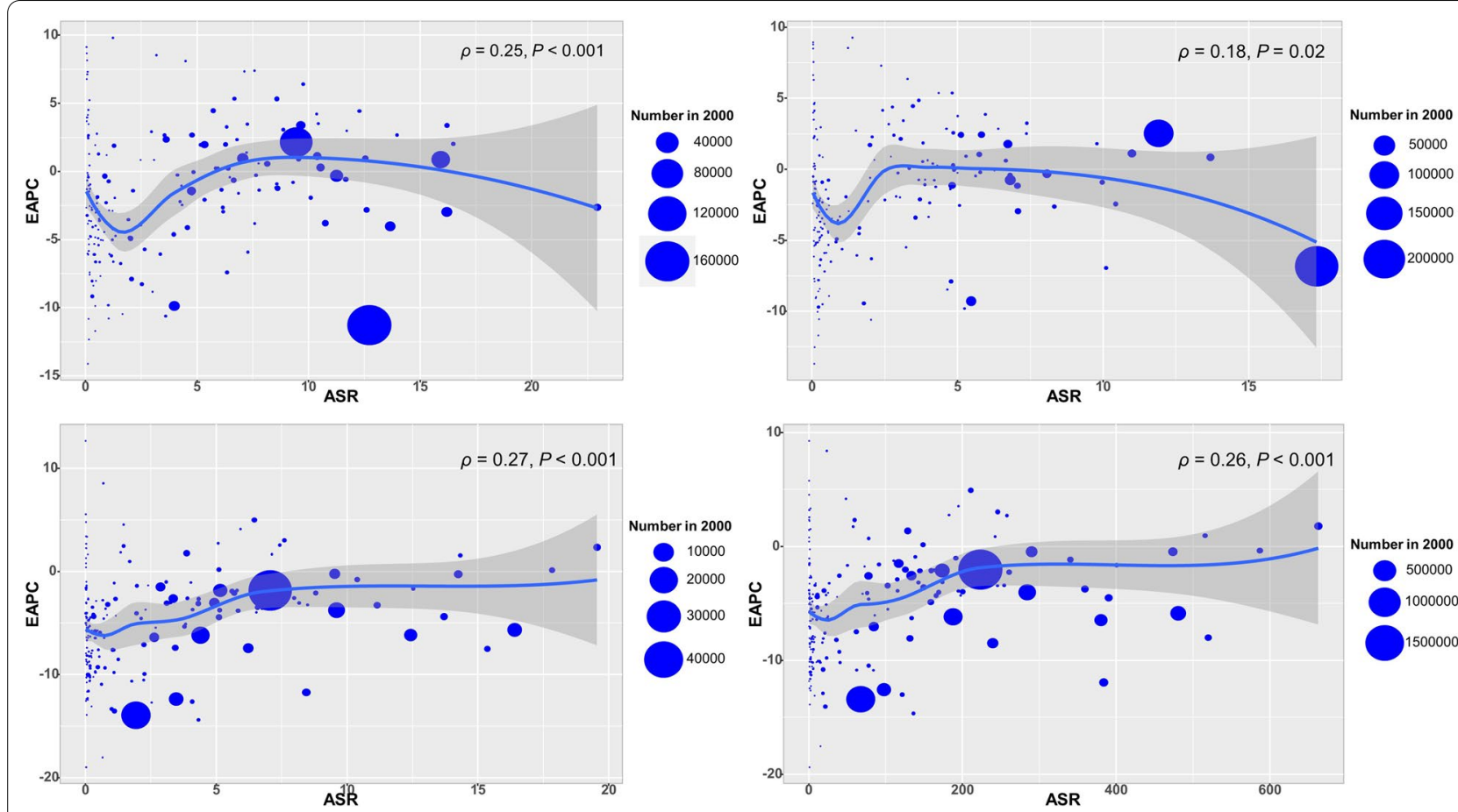

Fig. 4 The correlation between EAPCs (2000-2017) and ASR in 2000 at a national level. The EAPCs of MDR-TB burden including incidence (a), prevalence (b), death (c), and DALYS (d) had a positive correlation with the ASR in 2000, respectively. The correlation was calculated with Pearson correlation analysis. The size of circle increases with the numbers in 2000 in the corresponding countries. MDR-TB: multidrug resistant tuberculosis; ASR: Age-standardized rate; EAPC: Estimated annual percentage change; HDI: Human development index; DALYs: disability-adjusted life-years.

in 2017 at a national level $(\rho=0.27, P<0.001$, Fig. 4c; $\rho=-0.18, P=0.017$, Fig. 5a, respectively).

\section{Trends in MDR-TB-related DALYs}

Pronounced increasing trend was observed in DALYs associated with MDR-TB from 1990 to 1999, with an EAPC was 21.90 (95\% CI 16.55-27.50). The increasing trends occurred in all SDI areas and geographic regions. The largest rising trends of DALYs were observed in Central Asia $(\mathrm{EAPC}=62.87,95 \%$ CI 47.83-79.44), followed by Tropical Latin America, and South Asia (Additional file 1: Table S3; Fig. 1a). Rising trends of DALYs were observed in 163 countries/territories, with the most pronounced one being in Somalia $(\mathrm{EAPC}=79.93,95 \% \mathrm{CI}$ 70.87-89.47), followed by Turkmenistan, and Kyrgyzstan. However, seven countries had decreasing trends, particularly United States (EAPC $=-17.26,95 \% C I-19.91$ to - 14.54) (Additional file 1: Table S4; Figure S10A-C).

Globally, the number of DALYs due to MDR-TB was $4647.99 \times 10^{3}\left(95 \%\right.$ UI: $\left.2663.04 \times 10^{3}-7224.23 \times 10^{3}\right)$ in 2017, with a decrease of $17.71 \%$ from 2000 to 2107. The ASR of DALYs had a decreasing trend $(E A P C=-3.32$, 95\% CI-3.59 to-3.06) (Additional file 1: Table S3; Fig. 1b). During 2000 and 2017, the downward trends of
DALYs were observed in SDI areas and regions, expect Oceania and Australasia. The largest decreasing trends in DALYs were observed in East Asia and high-income Asia Pacific, in which the EAPCs were - 13.02 (95\% CI-13.58 to -12.46$)$ and -12.62 (95\% CI-13.85 to -11.38$)$, respectively (Additional file 1: Table S2; Fig. $1 \mathrm{~b}$, and Additional file 1: Figure S3A-C). At national level, the downward trends occurred in 164 countries/territories, with the largest decreasing trend being in Slovenia, Maldives, and Laos, in which the EAPCs were -19.35 (95\% $C I-21.10$ to -17.55$),-17.50 \quad(95 \% \quad C I-18.33$ to -16.66$)$, and -14.63 (95\% CI-15.65 to -13.59$)$, respectively. While upward trends were observed in nineteen countries/territories, with the largest one being in Singapore (EAPC $=9.28,95 \%$ CI 7.78-10.79), followed by Papua New Guinea and Zimbabwe (Additional file 1: Table S5; Figure S6A-C). The EAPCs (2000-2017) had a positive correlation with the ASR of DALYs in 2000, and a negative correlation with the HDI in 2017 at a national level $(\rho=0.26, P<0.001$, Fig. $4 d ; \rho=-0.16, P=0.029$, Fig. 5b, respectively). 


\section{Discussion}

In present study, the authors observed that the ASR of MDR-TB presented a parabolic distribution during the period 1990-2017, which peaked around 1999, and the lowest values were in 1990 and in 2017, respectively. For example, the largest overall ASIR were 6.41 in 1999, and the lowest values were 1.12 in 1990 and 5.55 in 2017, respectively. Therefore, 1999 was selected as the time cut-off point to describe its trends in two time periods, including 1990-1999, and 2000-2017. The ASR of MDRTB burden, including incidence, prevalence, death, and DALYs, dramatically increased globally between 1990 and 1999, but showed decreasing trends from 2000 to 2017, which objectively reflected the changing trends.

During 1990-1999, the absolute number of MDR-TB rapidly rose globally, and the ASR had the largest increasing trends in the areas of low and low-middle SDI areas. In these areas, there existed many stumbling blocks to TB control including population expansion, poverty, and overloaded health systems $[19,20]$. These factors could also explain why the EAPCs were negatively associated with HDI. Among geographic regions, central Asia showed the largest increasing trends of MDR-TB from 1990 to 1999. TB patients living in former Soviet countries had a high risk of MDR-TB development [21], probably attributed to a transmissible branch of the $M$. tuberculosis Beijing genotype: central Asia outbreak clade [22]. Somalia, Turkmenistan, and Kyrgyzstan had the most pronounced increasing trends in the ASR of MDRTB from 1990 to 1999. In these countries, poverty, malnutrition and poor health infrastructure are considerable barriers to health and wellbeing [23, 24]. Furthermore, the situation has been accelerated by an inundation of drug use and human immunodeficiency virus (HIV) infection $[25,26]$. Whereas a decreasing trend of MDRTB was observed in High-income North America, where the robust healthcare systems could treat TB effectively, and consequently to reduce the development of MDR-TB [27].

However, the decreasing trends of MDR-TB occurred in countries, regions and worldwide from 2000 to 2017, which was probably due to effective management and control of TB in recent years. The global tuberculosis report 2018 revealed that TB incidence was falling at about $2 \%$ per year, and the overall reduction of mortality rate during $2000-2017$ was estimated to be $42 \%$ [28]. The testing, detection and treatment of MDR-TB had achieved apparent progress globally, for example, up to $41 \%$ of TB patients were tested for rifampicin resistance in 2017 [29], and recommendations on the treatment and care of drug-resistant tuberculosis has been compiled and issued by WHO from 2011 to 2019[30]. Meanwhile, management of MDR-TB contacts had been developed

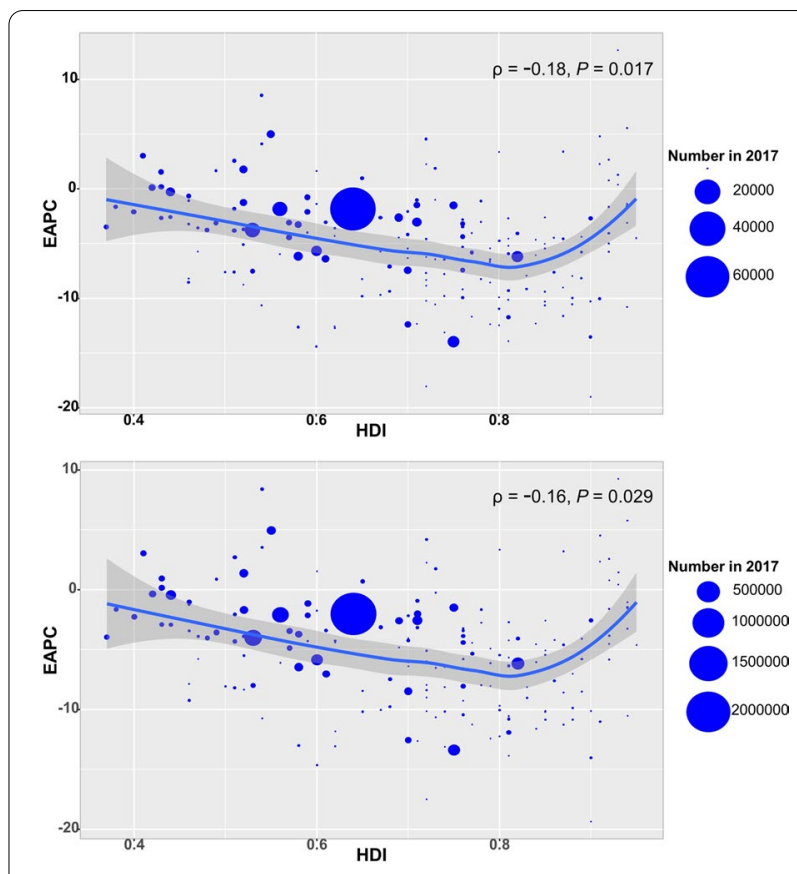

Fig. 5 The correlation between EAPCs (2000-2017) and HDI in 2017 at a national level. a The EAPCs of death due to MDR-TB had a negative correlation with the HDI in 2017; $\boldsymbol{b}$ The EAPCs of DALYS due to MDR-TB had a negative correlation with the HDI in 2017. The correlation was calculated with Pearson correlation analysis. The circles represent countries that were available on HDI data, and the size of circle increases with the numbers in 2017 in the corresponding countries. MDR-TB: multidrug resistant tuberculosis; ASR: Age-standardized rate; EAPC: Estimated annual percentage change; HDI: Human development index

in recent years, and the preventive therapy (TPT) could reduce the risk to development of MDR-TB by up to $90 \%$ [31]. Particularly, governments and political organizations have taken action for DR-TB control through action plans, financial initiatives, and health infrastructure worldwide [32, 33]. Pronounced decreasing trends of MDR-TB were found in Slovenia, Laos, and China. Due to the effective TB prevention and therapeutic programs, the combined MDR-TB prevalence was $1 \%$ or less in Slovenia [34]. Meanwhile, the efficient managements of TB were implemented in Slovenia, and the completeness of TB notification was as much as $100 \%$ [35]. Benefiting from the United Nation's Sustainable Development Goals (SDGs), the universal health coverage index had been substantially improved in Laos during the period 2000 2016 [36], which facilitated the control of TB. China was one of the countries with the largest population of TB in the world. The Chinese government started to revitalize anti-TB programs in 1990s, and carried out forceful measures to achieve considerable successes in MDR-TB control [37], which could provide a good example to other developing counties. A meta-analysis confirmed 
that the prevalence of MDR-TB had a decreasing trend in China from 1996 to 2014 [38]. Simultaneously, the government carried out national initiatives, including reducing poverty, improving health infrastructure, and implementing a new medical-care system in rural areas [39]. Surprisingly, several countries with a low incidence of tuberculosis exhibited obvious increasing trends of MDR-TB from 2000 to 2017, including Singapore, Australia and Papua New Guinea. These countries hold the most frequent population migration all over the world. In Singapore, $80 \%$ of MDR-TB patients occurred among the foreign population during 2000-2010, with an increasing trend after 2004 [40]. Papua New Guinea has a high TB burden, and causes challenges to cross-border management of MDR-TB in the Torres Strait, Australia [41]. Therefore, the importance of pre-immigration screening to detect the infectious disease should be emphasized [42].

Our study had three main limitations. First, estimation of disease burden was dependent mainly on the quality and quantity of data, and the accuracy and robustness of GBD estimates may have been impaired by a potential bias, including unreported cases, incomplete testing and reporting, and the test technology of MDR-TB varied across countries and over time. However, the GBD tuberculosis collaborators applied various modelling methods to estimates for locations with sparse data. For example, in countries without vital registration data, the tuberculosis death was assessed using the verbal autopsy studies, which had proven to be credible and sensitive [43]. Second, because of the limitation of ASR estimates, the trend of MDR-TB in age groups only were presented using percentage changes in absolute numbers. Third, the risk factors and clinical information of MDR-TB were not available, so the reason for the changing trends could not be investigated further.

\section{Conclusions}

The trends of MDR-TB burden dramatically increased during the period 1990-1999, but decreased from 2000 to 2017, indicating that the current control strategies are effective and feasible. However, the MDR-TB burden remains a substantial challenge to the public health globally, and more efficient strategies and increasing investment to the prevention and control of MDR-TB are required.

\footnotetext{
Abbreviations

DALYs: Disability-adjusted life-years; GBD: Global burden of disease; SDGs: Sustainable development goals; EAPC: Estimated annual percentage change; ASR: Age-standardized rate; Cl: Confidence interval; UI: Uncertainty interval; SDI: Socio-demographic index; HDI: Human development index; MDR-TB: Multidrug-resistant tuberculosis; TPT: The preventive therapy.
}

\section{Supplementary Information}

The online version contains supplementary material available at https://doi. org/10.1186/s40249-021-00803-w.

Additional file 1. The supplementary figures and tables of trends in MDRTB from 1990 to 2017.

\section{Acknowledgements}

Not applicable.

\section{Authors' contributions}

Z-JO: Project administration and drafting. D-FY, Y-XM, Y-HG: Data analysis and validation. Y-HL, H-SX: Data analysis and visualization. W-QH, Y-ZL, M-YZ, FW, $H H$ : Data collection and collation. QC: supervision and drafting and editing. All authors read and approved the final manuscript.

\section{Funding}

No funding was disclosed.

\section{Availability of supporting data}

All data during this study are included in this published article and its additional files.

Ethical approval and consent to participate

Not applicable.

\section{Consent for publication}

All authors consent for publication of the manuscript.

\section{Competing interests}

The authors declare no competing interests.

\section{Author details}

${ }^{1}$ Guangdong Provincial Key Laboratory of Tropical Disease Research, Department of Epidemiology, School of Public Health, Southern Medical University, 1838 Guangzhou North Road, Guangzhou 510515, China. ${ }^{2}$ Department of MICU, Guangdong Women and Children Hospital, Guangzhou, China.

Received: 21 August 2020 Accepted: 4 February 2021

Published online: 06 March 2021

\section{References}

1. Nathanson E, Nunn P, Uplekar M, Floyd K, Jaramillo E, Lonnroth K, et al. MDR tuberculosis-critical steps for prevention and control. N Engl J Med. 2010;363(11):1050-8.

2. Mariandyshev A, Eliseev P. Drug-resistant tuberculosis threatens WHO's End-TB strategy. Lancet Infect Dis. 2017;17(7):674-5.

3. Sharma SK, Mohan A. Multidrug-resistant tuberculosis: a menace that threatens to destabilize tuberculosis control. Chest. 2006;130(1):261-72.

4. Pradipta IS, Forsman LD, Bruchfeld J, Hak E. Risk factors of multidrugresistant tuberculosis: A global systematic review and meta-analysis. J Infect. 2018:77(6):469-78.

5. Van der Werf MJ, Langendam MW, Huitric E, Manissero D. Multidrug resistance after inappropriate tuberculosis treatment: a meta-analysis. Eur Respir J. 2012;39(6):1511-9.

6. Frieden TR, Sterling T, Pablos-Mendez A, Kilburn JO, Cauthen GM. The emergence of drug-resistant tuberculosis in New York City. N Engl J Med. 1993;328(8):521-6.

7. Monno L, Angarano G, Carbonara S, Coppola S, Costa D, Quarto M, et al. Emergence of drug-resistant Mycobacterium tuberculosis in HIV-infected patients. Lancet. 1991:337(8745):852

8. Dheda K, Gumbo T, Maartens G, Dooley KE, McNerney R, Murray M, et al. The epidemiology, pathogenesis, transmission, diagnosis, and management of multidrug-resistant, extensively drug-resistant, and incurable tuberculosis. Lancet Respir Med. 2017;5(17):291-360. 
9. Falzon D, Desenclos JC. World TB day: European countries report over 400,000 tuberculosis cases in 2004. Euro Surveill. 2006;11(3):E0603233.

10. Pablos-Mendez A, Raviglione MC, Laszlo A, Binkin N, Rieder HL, et al. Global surveillance for antituberculosis-drug resistance, 1994-1997: World Health Organization-International Union against Tuberculosis and Lung Disease Working Group on Anti-Tuberculosis Drug Resistance Surveillance. N Engl J Med. 1998;338(23):1641-9.

11. WHO. Global tuberculosis report 2017. Geneva: World Health Organization. https://www.who.int/teams/global-tuberculosis-programme/tbreports. Accessed 26 Oct 2017.

12. Zignol M, van Gemert W, Falzon D, Sismanidis C, Glaziou P, Floyd K, et al. Surveillance of anti-tuberculosis drug resistance in the world: an updated analysis, 2007-2010. Bull World Health Organ. 2012;90(2):111-9.

13. Shah NS, Pratt R, Armstrong L, Robison V, Castro KG. Extensively drug-resistant tuberculosis in the United States, 1993-2007. JAMA. 2008;300(18):2153-60.

14. WHO. Global tuberculosis report 2014. Switzerland: World Health Organization. https://www.who.int/teams/global-tuberculosis-programme/ tb-reports. Accessed May 082014.

15. O'Donnell MR, Jarand J, Loveday M, Padayatchi N, Zelnick J, Werner L, et al. High incidence of hospital admissions with multidrug-resistant and extensively drug-resistant tuberculosis among South African health care workers. Ann Intern Med. 2010;153(8):516-22.

16. Mukund U, Diana W, Knut L, Ernesto J, Christian L, Hannah M, et al. WHO's new end TB strategy. Lancet. 2015;385(9979):1799-801.

17. Reid MJA, Arinaminpathy N, Bloom A, Bloom BR, Boehme C, Chaisson $R$, et al. Building a tuberculosis-free world: The Lancet Commission on tuberculosis. Lancet. 2019;393(10178):1331-84.

18. Liu Z, Jiang Y, Yuan H, Fang Q, Cai N, Suo C, et al. The trends in incidence of primary liver cancer caused by specific etiologies: Results from the Global Burden of Disease Study 2016 and implications for liver cancer prevention. J Hepatol. 2019;70(4):674-83.

19. Marais BJ, Raviglione MC, Donald PR, Harries AD, Kritski AL, Graham $\mathrm{SM}$, et al. Scale-up of services and research priorities for diagnosis, management, and control of tuberculosis: a call to action. Lancet. 2010;375(9732):2179-91.

20. Zhao P, Li XJ, Zhang SF, Wang XS, Liu CY. Social behaviour risk factors for drug resistant tuberculosis in mainland China: a meta-analysis. J Int Med Res. 2012;40(2):436-45.

21. Falzon D, Infuso A, F A-B. In the European Union, TB patients from former Soviet countries have a high risk of multidrug resistance. International Journal of Tuberculosis and Lung Disease. 2006;10(9):954-8.

22. Shitikov E, Vyazovaya A, Malakhova M, Guliaev A, Bespyatykh J, Proshina E, et al. Simple Assay for Detection of the Central Asia Outbreak Clade of the Mycobacterium tuberculosis Beijing Genotype. J Clin Microbiol. 2019:57(7):215-9.

23. Menon S. Preventing nosocomial MDR TB transmission in sub Saharan Africa: where are we at? Glob J Health Sci. 2013;5(4):200-10.

24. Wright A, Zignol M, Van Deun A, Falzon D, Gerdes SR, Feldman K, et al. Epidemiology of antituberculosis drug resistance 2002-07: an updated analysis of the Global Project on Anti-Tuberculosis Drug Resistance Surveillance. Lancet. 2009;373(9678):1861-73.

25. Schluge NW, El-Bassel N, Hermosilla S, Terlikbayeva A, Darisheva M, Aifah A, et al. Tuberculosis, drug use and HIV infection in Central Asia: an urgent need for attention. Drug Alcohol Depend. 2013;132(Suppl 1):S32-6.

26. Harries AD, Hargreaves NJ, Kemp J, Jindani A, Enarson DA, Maher D, et al. Deaths from tuberculosis in sub-Saharan African countries with a high prevalence of HIV-1. Lancet. 2001;357(9267):1519-23.
27. CfDCa P. Trends in tuberculosis - United States, 2011. MMWR Morb Mortal Wkly Rep. 2012;61(11):181-5.

28. WHO. Global tuberculosis report 2018. Geneva, Switzerland: World Health Organization. https://www.who.int/teams/global-tuberculosis-progr amme/tb-reports. Accessed September 202018

29. WHO. Process overview: development of a global strategy for TB research and innovation Geneva: World Health Organization. https://apps.who.int/ iris/handle/10665/336076. Accessed June 5 2019, 2019.

30. WHO Guidelines Approved by the Guidelines Review Committee. WHO consolidated guidelines on tuberculosis: Module 4: Treatment - Drugresistant tuberculosis treatment. Geneva. https://www.researchgate.net/ publication/343110810. Accessed July 2120202020.

31. Marks SM, Mase SR, SB M. Systematic Review, Meta-analysis, and Costeffectiveness of Treatment of Latent Tuberculosis to Reduce Progression to Multidrug-Resistant Tuberculosis. Clin Infect Dis. 2017;64(12):1670-7.

32. Abubakar I, Zignol M, Falzon D, Raviglione M, Ditiu L, Masham S, et al. Drug-resistant tuberculosis: time for visionary political leadership. Lancet Infect Dis. 2013;13(6):529-39.

33. Raviglione M, Marais B, Floyd K, Lonnroth K, Getahun H, Migliori GB, et al. Scaling up interventions to achieve global tuberculosis control: progress and new developments. Lancet. 2012;379(9829):1902-13.

34. Drobniewski FA, Balabanova YM. The diagnosis and management of multiple-drug-resistant-tuberculosis at the beginning of the new millenium. Int J Infect Dis. 2002;6(Suppl 1):S21-31.

35. Straetemans M, Bakker MI, Alba S, Mergenthaler C, Rood E, Andersen PH, et al. Completeness of tuberculosis (TB) notification: inventory studies and capture-recapture analyses, six European Union countries, 2014 to 2016. Euro Surveill. 2020;25:12.

36. Collaborators GS. Measuring progress and projecting attainment on the basis of past trends of the health-related Sustainable Development Goals in 188 countries: an analysis from the Global Burden of Disease Study 2016. Lancet. 2017;390(10100):1423-59.

37. Bhatter $\mathrm{P}, \mathrm{Chatterjee} \mathrm{A}$. The dragon and the tiger: realties in the control of tuberculosis. Interdiscip Perspect Infect Dis. 2012;2012:625459.

38. Zhang J, Gou H, Hu X, Hu X, Shang M, Zhou J, et al. Status of drug-resistant tuberculosis in China: A systematic review and meta-analysis. Am J Infect Control. 2016;44(6):671-6.

39. Zhang D, Shi L, Tian F. Care Utilization with China's New Rural Cooperative Medical Scheme: Updated Evidence from the China Health and Retirement Longitudinal Study 2011-2012. Int J Behav Med. 2016;23(6):655-63.

40. Chee CB, Khin-Mar KW, Cutter J. The imminent threat of multidrug-resistant tuberculosis in Singapore. Singapore Med J. 2012;53(4):238-40.

41. Francis JR, Manchikanti P, Blyth CC, Denholm J, Lowbridge C, Coulter C, et al. Multidrug-resistant tuberculosis in Australia, 1998-2012. Int J Tuberc Lung Dis. 2018;22(3):294-9.

42. Lowenthal P, Westenhouse J, Moore M, Posey DL, Watt JP. Reduced importation of tuberculosis after the implementation of an enhanced preimmigration screening protocol. Int J Tuberc Lung Dis. 2011;15(6):761-6.

43. GBD Tuberculosis Collaborators. The global burden of tuberculosis: results from the Global Burden of Disease Study 2015. Lancet Infect Dis. 2018:18(3):261-84.

Ready to submit your research? Choose BMC and benefit from:

- fast, convenient online submission

- thorough peer review by experienced researchers in your field

- rapid publication on acceptance

- support for research data, including large and complex data types

- gold Open Access which fosters wider collaboration and increased citations

- maximum visibility for your research: over 100M website views per year

At BMC, research is always in progress.

Learn more biomedcentral.com/submissions 